\title{
Criminologie
}

\section{La contribution féministe à l'étude de la déviance en criminologie}

\section{Colette Parent}

Volume 25, numéro 2, 1992

Nouvelles connaissances et nouvelles questions en criminologie

URI : https://id.erudit.org/iderudit/017323ar

DOI : https://doi.org/10.7202/017323ar

Aller au sommaire du numéro

Éditeur(s)

Les Presses de l'Université de Montréal

ISSN

0316-0041 (imprimé)

1492-1367 (numérique)

Découvrir la revue

Citer cet article

Parent, C. (1992). La contribution féministe à l'étude de la déviance en criminologie. Criminologie, 25(2), 73-91. https://doi.org/10.7202/017323ar
Résumé de l'article

Beginning in the 70s, the silence on the question of women in criminology was broken by a feminist series of works that was set upon the questioning and updating of knowledge in the social sciences and in criminology. This article concerns the conception of deviance in feminist studies dealing with the "criminality" and criminalization of women. The author finds that the feminists adopt a firmly empathetical approach toward women offenders, which enables them to depart from the traditional concepts of deviance and the develop analyses that go beyond the known context of questioning in the discipline. The feminist approach also makes it possible to review the positions to be taken regarding the crime policies that should be applied. 
Beginning in the 70s, the silence on the question of women in criminology was broken by a feminist series of works that was set upon the questioning and updating of knowledge in the social sciences and in criminology. This article concerns the conception of deviance in feminist studies dealing with the "criminality" and criminalization of women. The author finds that the feminists adopt a firmly empathetical approach toward women offenders, which enables them to depart from the traditional concepts of deviance and the develop analyses that go beyond the known context of questioning in the discipline. The feminist approach also makes it possible to review the positions to be taken regarding the crime policies that should be applied.

La première moitié des années soixante-dix marque un éclatement des orientations thériques et la naissance de points de vue alternatifs en sciences sociales et en criminologie. Cette même période se caractérise par l'introduction de productions criminologiques féministes qui s'alimentent à la fois aux réflexions et mouvements théoriques dans la discipline et aux apports féministes en dehors du champ criminologique.

Le silence sur la question des femmes fait place à une production d'abord modeste mais jalonnée d'un certain nombre d'œuvres importantes sur la "criminalité " (Bertrand, 1979; Heidensohn, 1968; Klein, 1973; Klein et Kress, 1976; Smart, 1976, etc.) et la victimation (Schwendinger, Herman et Julia, 1974, etc.) des femmes. En revanche, malgré un accroissement du nombre d'analyses féministes durant les années quatre-vingt, celles-ci demeurent peu reconnues, et leur effort sur la discipline plutôt faible. En 1988, Gelsthorpe et Morris constatent que le féminisme en criminologie est toujours considéré comme une activité fermée, isolée, comme le domaine d'un groupe spécifique de femmes.

Cette marginalisation ne freine toutefois pas la démarche féministe qui, au fil des années, poursuit sa quête vers l'élaboration d'un savoir sur la question des femmes. Si bien qu'en 1990 Smart affirme

1. Professeure, Département de criminologie, Université d'Ottawa, Ottawa, Ontario K1N 6N5. 
qu'aujourd'hui ce n'est peut-être pas tant le féminisme qui a besoin de la criminologie mais la criminologie qui a besoin du féminisme.

Il nous apparaît, en effet, que depuis les vingt dernières annés la production féministe participe du questionnement et du renouvellement du savoir en sciences sociales et plus particulièrement en criminologie ${ }^{2}$.

Nous n'avons pas la prétention de pouvoir dégager ici toutes les dimensions de cette contribution, mais nous entendons présenter la conception féministe de la "criminalité » et la criminalisation des femmes, et les conséquences qu'elle entraîne pour ce qui est des politiques criminelles. Notre analyse s'appuie sur un corpus de textes féministes portant sur la «criminalité» et la criminalisation des femmes, produits depuis les années soixante-dix et constituant un apport face aux questionnements théoriques dans la discipline ${ }^{3}$. Avant d'aller plus loin, précisons que nous définissons le féminisme occidental contemporain comme un mouvement social qui vise la libération des femmes et comme une tendance intellectuelle (aux perspectives multiples) qui porte sur l'analyse des rapports sociaux de sexe ou, tout au moins, sur la subordination, la domination des femmes.

L'approche féministe donne alors aux auteurs qui se penchent sur la problématique de la "criminalite » et la criminalisation des femmes un éclairage déterminé; leur point de départ, et leur fil conducteur, en est un résolument empathique envers les femmes justiciables, vues davantage comme des sœurs dans l'oppression que des ennemies de la société ou encore des personnes malades. Ces analyses seront donc susceptibles d'apporter des changements dans la conception traditionnelle de la déviance et dans les propositions de politiques criminelles.

\section{LES ANALYSES SUR LA «CRIMINALITÉ» DES FEMMES}

La contribution initiale des analyses féministes repose sur la critique des théories traditionnelles sur la «criminalité" des femmes. Des analyses pionnières comme celles de Klein (1973) et Smart (1976) se sont d'abord et avant tout donné comme objectif de mettre à jour les

2. Pour une analyse des grandes questions soulevées par les analyses féministes sur la «criminalité» et la criminalisation des femmes, voir Laberge (1991). Pour une vision générale de la contribution féministe au renouvellement de la discipline, voir Gelsthorpe et Morris (1990).

3. Pour une recension de l'ensemble des travaux sur lesquels s'appuie cette analyse, voir Parent (1992). 
stéréotypes sexistes qui alimentent ces théories. Elles ont aussi fait ressortir, dans une certaine mesure, les limites d'une criminologie positiviste dont les prémisses sont inadéquates, et qui se présente comme un outil de contrôle et de préservation du statu quo.

Mais leur contribution dépasse ces volets qui ne constituent, tant pour Smart que pour Klein, que la première étape de la production d'un savoir féministe en criminologie. Après avoir compris les bases sur lesquelles repose le savoir criminologique sur les femmes, il sera possible, selon Klein (1973), de concevoir un autre type de recherche appuyé sur une nouvelle conception de la «criminalité» des femmes, des individus et de leur rapport à l'État. Smart (1976), quant à elle, considère que cette première étape ne permet pas de produire une nouvelle théorie sur la «criminalité» des femmes. Pour répondre aux silences de la criminologie sur les femmes, il faut réaliser d'autres recherches. Mais ici la tâche est complexe, et Smart (1976) rappelle qu'autant dans les sciences que dans la société en général les femmes ont êté définies comme l'Autre, l'inessentielle (De Beauvoir, 1949); il ne suffit donc pas d'accumuler des informations sur les femmes dans la justice pénale pour corriger cet état de fait. En effet, pour pallier le caractère, non seulement partiel, mais aussi partial de la criminologie, Smart $(1976,180)$ indique qu'il nous «faut trouver des voies alternatives pour conceptualiser l'univers social de sorte que les intérêts et les préoccupations des femmes soiènt considérés et inclus plutôt que subsumés ou ignorés ${ }^{4}$ ".

La tâche des féministes, du moins celles qui suivent les pas de Klein (1973) et de Smart (1976), ne se limite donc pas à intégrer la problématique des femmes dans la justice pénale aux problématiques existantes, elle vise du même coup la construction d'un savoir criminologique sur les femmes, non plus comme un addendum à celui des hommes, mais comme un savoir issu de l'univers social même des femmes.

Le cheminement féministe exigeait donc une prise de distance critique des analyses criminologiques traditionnelles. En revanche, cette recherche n'a pas été sans avatars, et c'est à des degrés fort différents que les analyses féministes sur la «criminalité » des femmes ont su mettre en cause les fondements de la criminologie et de la politique criminelle traditionnelles.

4. Notre traduction. 
D'abord, la thèse de la relation entre le mouvement de libération des femmes et la hausse de leur taux de «criminalité», proposée par Adler (1975) et Simon (1975), a suscité beaucoup d'intérêt, et les recherches sur ce thème se poursuivent encore aujourd'hui. Mais force est de reconnaître que la thèse du lien entre les rôles sexuels et la criminalité remonte au XIX ${ }^{e}$ siècle et que celle-ci s'inscrit d'emblée dans une perspective traditionnelle qui ne met pas en cause la notion de crime et axe la recherche sur la différence entre criminels et non-criminels. Bien que les auteures aient tenté d'appuyer leur analyse sur une perspective féministe libérale, on n'en constate pas moins que leur thèse présente potentiellement plus d'intérêt pour les agences de contrôle social (préoccupées de combattre le crime) que pour les féministes soucieuses de venir en aide aux femmes justiciables. Des auteures féministes ont d'ailleurs souligné qu'elles pouvaient contribuer à discréditer le mouvement de libération des femmes en suggérant que celui-ci pouvait entrainer des femmes à commettre des illégalismes, à devenir «criminelles " au même titre que les hommes ${ }^{5}$.

D'autres criminologues féministes, préoccupées de répondre au caractère partiel de la discipline, se sont penchées sur les théories criminologiques sociologiques sur la «criminalité» des hommes pour vérifier l'application que l'on en fait aux femmes. Mais cette recherche a mis davantage en évidence que la discipline n'avait pas seulement produit un savoir partiel, rendant compte uniquement du monde des hommes, mais aussi partial, c'est-à-dire un savoir produit à partir de la perspective des hommes. En somme, comme l'ont fait ressortir Leonard (1982) et Naffine (1987), ce type de démarche s'est heurté au sexisme qui a cours même en ce qui a trait à l'élaboration des connaissances en sciences sociales et en criminologie et qui, là comme ailleurs, a contribué à modeler les théories à la source même. Appliquer, sans autre forme d'analyse critique, ces théories aux femmes ne peut que consolider le savoir masculin à la base de la discipline.

D'autres réflexions, dont celles de Carlen (1988), de Laprairie (1987) et de Messerschmidt (1986), se sont plutôt orientées vers l'élaboration de nouvelles théories qui rendent compte précisément de la «criminalité » des femmes. Ces analyses marquent une plus grande distance du paradigme traditionnel. Bien qu'elles maintiennent la recherche des causes du comportement criminel et qu'en général elles ne mettent pas en question la notion de crime, elles ne considèrent plus les

5. Cette thèse a d'ailleurs fait l'objet de critiques nombreuses et fort nouries. Voir à ce sujet, entre autres, Box (1983), Heidensohn (1985) et Smart (1979). 
caractéristiques biologiques ou psychologiques des femmes, mais plutôt les facteurs politiques, économiques et sociaux où s'inscrit leur «criminalité». À partir de l'univers social des femmes, les analyses nous révèlent enfin les rapports de pouvoir que vivent ces dernières; elles présentent leurs comportements de transgression comme ceux d'un groupe sans pouvoir, victimes d'une société sexiste, voire raciste, et fondée sur des rapports de classe. Ce type d'analyse n'est donc pas accompagné de propositions de changement au service des institutions de contrôle avec des objectifs de réhabilitation ou de neutralisation; il est plutôt associé à des propositions de réformes qui visent à donner du pouvoir aux femmes en général et aux femmes justiciables en particulier.

La réflexion de Carlen nous semble être celle qui pousse le plus loin dans cette voie, qui rompt de façon significative avec la conception traditionnelle de la déviance. D'abord, elle récuse toute possibilité d'élaborer une théorie générale de la «criminalité » ou même une théorie propre à la «criminalité » des femmes (Carlen, 1985). Selon elle, la "femme criminelle» n'existe pas; les femmes qui entrent en conflit avec la loi viennent de tous les milieux socio-culturels, bien que ce soient surtout celles qui viennent des milieux les plus défavorisés qui, comme les hommes, sont prises en charge par le système pénal. En revanche, Carlen ne renonce pas à toute forme d'analyse des situationsproblèmes et propose une thérie explicative du comportement de transgression et de la criminalisation des femmes de classes defavorisées qui se sont engagées dans une «carrière » déviante (Carlen, 1988). Inspirée d'une analyse ethnographique de l'expérience de vie et de la criminalisation de 39 femmes dont elle a recueilli les récits de vie, elle constate que le peu d'options de vie sociale offertes à ces femmes dans nos sociétés capitalistes et patriarcales, jointes à leur adhésion aux valeurs de consommation et leur quête pour une vie décente constituent des éléments qui peuvent les entraîner vers des comportements illégaux. Lorsque cela se produit, la réaction sociale contribue à réduire encore davantage leurs options sociales et peut les amener à choisir une «carrière " déviante plutôt que d'être acculées à l'isolement et à la pauvreté. En somme, Carlen constate que les conditions matérielles de ces femmes dans nos sociétés patriarcales et capitalistes et le sentiment d'injustice sociale qu'elles éprouvent les amènent à opter peu à peu pour les illégalismes. Elle associe donc étroitement comportement illégal et réaction sociale, et conçoit la nécessité de comprendre la problématique des femmes justiciables à partir de l'expérience de vie propre à des groupes de femmes aux prises avec la justice. 
Pour pallier cette situation, Carlen propose une série de mesures qui permettent au contraire d'élargir leurs options de vie sociale. Elle suggère des réformes partielles dans différentes institutions et des changements structuraux et idéologiques fondamentaux susceptibles, entre autres, de réduire la dépendance des femmes.

Les théories propres à la "criminalité» des femmes permettent donc de dégager les facteurs structuraux dans lesquels s'inscrivent les comportements problématiques des femmes qui ont des démêlés avec la justice et mettent en lumière la dimension du contrôle dans la production du «crime». Même si, en général, elles ne mettent pas précisément en cause la notion de crime, elles complexifient cette problématique qui ne saurait se réduire à une inadéquation individuelle, qu'elle soit de nature biologique, psychologique ou sociale. Du même coup, ces analyses prennent leurs distances par rapport aux objectifs utilitaires qui marquent les analyses criminologiques traditionnelles et apparaissent au service des femmes justiciables et des femmes en général.

Dans l'ensemble, il nous est apparu que les analyses féministes sur le comportement de transgression des femmes ont surtout contribué à faire ressortir le caractère partial de la criminologie pensée d'abord et avant tout par et pour les hommes. Certaines ont aussi apporté un éclairage différent sur le comportement des femmes justiciables en l'analysant à partir de leur contexte biographique et en l'associant aux conditions socio-politico-économiques qui affectent un nombre important de femmes dans nos sociétés. Leurs études ont permis de déboucher sur des propositions de changements institutionnels et structuraux afin de contrer la situation de moindre pouvoir des femmes justiciables.

Mais les féministes ont aussi tourné leur regard vers la criminalisation des femmes et, en s'inspirant des perspectives interactionnistes et critiques, elles contribuent également à redéfinir la conception de la déviance.

\section{LA CONTRIBUTION INTERACTIONNISTE À L'ÉTUDE DU COMPORTEMENT}

La production interactionniste sur les femmes, à l'instar des analyses traditionnelles de la discipline, s'est révélée partielle et partiale ${ }^{6}$. Malgré la richesse de cette approche, ce constat s'applique à la fois à la première vague d'études phénoménologiques de l'École de Chicago

6. Nous empruntons l'expression à Juteau-Lee (1981). 
(1920-1945) et au deuxjème volet d'analyses sur la réaction sociale (après 1960 tout particulièrement).

\subsection{Les études phénoménologiques}

On peut bien sûr faire état de l'apport de Thomas, précurseur de l'interactionnisme, dans The Unadjusted Girl, publié en 1923. Celui-ci nous présente un portrait fort empathique de la situation des jeunes filles qui vendent des services sexuels et sait faire ressortir les souffrances qui peuvent y être associées. Mais son analyse s'appuie sur les conceptions traditionnelles de la nature et du rôle des femmes, et reconduit le double standard de moralité selon le sexe. À sa suite, la première vague d'études de l'École de Chicago a fait silence sur la question des femmes: un silence qui se poursuit jusque dans les années quatre-vingt, alors que des auteures féministes font appel aux histoires de la vie de ces femmes et adoptent ainsi une démarche analogue à certains égards à celle de l'École de Chicago.

En effet, appuyées sur l'approche phénoménologique, quelques chercheures ont étudié le vécu des jeunes filles et des femmes aux príses avec le système de justice criminelle. D'autres ont conçu des analyses d'après différentes approches pour permettre aux femmes de rendre compte de leur insertion dans la société à partir d'elles-mêmes. Du quotidien des jeunes femmes qui se dégage à la suite de l'approche phénoménologique, elles ont pu intégrer des réflexions sur les dimensions structurelles de leur vécu.

Citons ici en exemple l'étude de Blom et de van den Berg (1989) qui nous offrent une typologie de la vie et des modes de travail des prostituées héroïnomanes. Les deux sociologues avaient comme objectifs initiaux d'étudier le processus d'entrée en prostitution et la sousculture des prostituées afin de pouvoir expliquer le faible taux de participation des jeunes filles aux programmes de traitement offerts. Mais, une fois sur le terrain, ces objectifs seront adaptés à la complexité de la problématique. $D^{\star}$ abord, les sociologues prennent la décision de mener une recherche non pas ethnographique, comme conçue initialement, mais plutôt biographique ; ensuite, ils abandonnent le concept de sous-culture parce que trop étroit pour rendre compte du monde complexe de la prostitution à Amsterdam. Ils décident de féminiser le modèle de carrière choisi de manière à rendre compte de façon précise des transitions de carrière et des comportements reliés aux rôles sexuels chez les prostituées hérö̈nomanes. La recherche permet en effet de constater que la plupart des transitions de carrière des jeunes filles sont reliées à des dimensions de leurs relations personnelles avec des amants 
ou amis proches. Elle permet aussi de dégager deux grandes dimensions de leurs relations avec leur «ami », soit l'autonomie versus la subordination et les associations sexuelles inspirées par l'attachement romantique ou les intérêts d'affaires. De ce fondement, le chercheur et la chercheure établissent une typologie des prostituées héroïnomanes constituée à partir de trois variables: d'abord la famille d'origine où sont considérées les dimensions de classe, ensuite les liens personnels où se fait sentir l'influence du quotidien et, finalement, le type de rôle sexuel adopté où les relations de sexe marquent fortement leur influence. Cette typologie leur permet d'expliquer «les différentes routes choisies par les prostituées héroïnomanes et leur réponse aux programmes de traitement offerts ${ }^{7} »$. Nous retrouvons alors une recherche fondée sur les histoires de vie, pensée pour servir les sujets elles-mêmes et qui présente une analyse où sont prises en compte les dimensions structurelles affectant leur vie. Pour terminer, cette étude offre des solutions en matière de traitements pour les jeunes prostituées hérönomanes à partir de leurs besoins, et ce, sans recourir à la voie trop bien connue qu'est la coercition.

Les analyses phénoménologiques comme celles de Blom et de van den Berg (1989) permettent de dépasser plusieurs limites du paradigme traditionnel et d'orienter la réflexion vers des voies nouvelles. D'abord, elles contribuent à éclairer la question des femmes dans la discipline en articulant la recherche à partir du monde des femmes et de leurs expériences de vie. Ensuite, elles abordent la dimension du comportement à partir de la perspective des femmes elles-mêmes et non pas de la distante et illusoire recherche de la différence entre «criminels» et non-«criminels». Plus encore, elles articulent la dimension du comportement aux dimensions structurelles qui affectent la vie des femmes et permettent ainsi de mieux appréhender la complexité et la diversité des expériences de vie des femmes qui entrent en contact avec le système de justice criminelle. Cette prise en compte jointe à la partialité critique envers les femmes, caractéristique des analyses féministes, donne les conditions pour dépasser à la fois le caractère utilitaire des politiques criminelles proposées dans le cadre du paradigme traditionnel et le silence des interactionnistes sur les politiques sociales à mettre de l'avant. Les propositions de changement peuvent donc être adaptés aux intérêts et besoins des femmes elles-mêmes, sujets d'analyse. Enfin, ces études présentent une démarche de recherche qui rompt avec le modèle positiviste en criminologie.

7. Notre traduction. 


\subsection{Les études sur la réaction sociale}

La seconde vague d'études de l'École de Chicago qui a porté sur la réaction sociale à la déviance n'a guère été plus productive à propos de la question des femmes. Nous nous devons de souligner ici l'intéressante étude interactionniste de Prudence Rains (1971) sur la carrière morale de la jeune mère célibataire. Mais la publication de la première œuvre interactionniste complète sur la problématique des femmes dans la justice pénale par E.M. Schur remonte à 1984. Subséquente aux critiques féministes adressées à l'interactionnisme, cette œuvre tente d'y répondre et s'inspire d'ailleurs fortement des productions féministes en sciences sociales.

L'analyse de Schur (1984) nous introduit aux processus d'étiquetage, de stigmatisation que subissent, à des degrés divers, toutes les femmes. L'auteur constate, en effet, que d' «une façon ou d'une autre, presque toutes les femmes dans notre société sont affectées par les définitions dominantes de la déviance" puis articule son analyse autour de la proposition théorique suivante: être femme, c'est occuper un statut déviant (Schur, 1984, p. 3). Il nous montre alors que les hommes représentent le standard de normalité dans nos sociétés et dévoile la situation de moindre pouvoir qui affecte toutes les femmes, à différents degrés. La déviance des femmes nous est prêsentée comme un construit social, et Schur (1984) analyse tant les formes de contrôles informels que formels exercés sur elles à partir de ce contexte global de moindre pouvoir. Sa démarche nous permet enfin de pénétrer la dimension du contrôle social exercé à l'égard des femmes non plus comme une simple réponse au comportement, mais comme le résultat d'un processus de définition sociale. Il permet également d'échapper aux limites traditionnelles de l'interactionnisme, incapable de reconnaître les rapports de pouvoir entre les hommes et les femmes dans les interactions quotidiennes et dans la production de la déviance. De plus, cette analyse laisse place à l'hypothèse que l'ensemble des normes imposées précisément aux femmes font partie, du moins dans une certaine mesure, des paramètres d'intervention auprès des femmes dans la justice pénale et influe sur le traitement auquel elles sont soumises. Notons finalement que, si Schur (1984, p. 19-20) ne propose pas de changements précis dans le domaine des rapports sociaux de sexe, il souhaite que les femmes acquièrent plus de pouvoir dans nos sociétés et considère que son analyse peut constituer un prérequis au changement social. Là encore, la recherche féministe interactionniste permet de dépasser à la fois les limites du paradigme traditionnel et de l'approche interactionniste. 
Les emprunts féministes à l'approche interactionniste ont donc permis d'explorer de nouvelles avenues dans l'étude de la déviance en criminologie. Les analyses féministes sur la criminalisation des femmes ont à leur tour apporté leur contribution.

\section{LES ANALYSES SUR LA CRIMINALISATION DES FEMMES}

Dès la fin des années soixante-dix, en effet, nous retrouvons des recherches féministes qui mettent l'accent non plus sur le comportement criminel, mais sur la réaction sociale; elles orientent alors l'analyse vers l'État et les appareils d'État dans la création et l'application des lois, et présentent des analyses sur le droit comme outil de discrimination et/ou de lutte (Smart, 1986, etc.), sur l'élaboration d'un droit féministe (Heidensohn, 1986, etc.), sur le traitement sexiste des femmes dans la justice pénale, (Daly, 1987; Parent, 1986, etc.). Dans les années quatre-vingt, nous retrouvons aussi des études qui portent sur la déconstruction du droit (Smart, 1989) et sur la construction de la féminité dans nos sociétés capitalistes (Sumner, 1990) et plus particulièrement dans le domaine de l'appareil pénal (Eaton, 1986; Worrall, 1990, etc.).

Cependant, le comportement criminel n'est pas toujours évacué de l'analyse: dans certains cas, l'approche théorique s'appuie précisément sur une position dualiste considerant à la fois le comportement déviant et le processus de criminalisation ; c'est le cas, entre autres, des travaux marxistes-féministes (Klein et Kress, 1976; Rafter et Natalazia, 1981) et, plus tard, féministes-socialistes (Currie, 1986; Gregory, 1986). Dans d'autres cas, l'analyse emprunte de façon assez aléatoire à l'une ou l'autre dimension. Bien que ces travaux fassent encore état de la dimension du comportement, ils portent d'abord et avant tout sur les contrôles exercés à l'égard des femmes (Dahl et Snare, 1978; Leonard, 1982).

En effet, en tournant leur regard vers le traitement des femmes dans la justice pénale, nombre d'études féministes ont inđiqué la nécessité de situer cette problématique dans le cadre plus général de la situation des femmes dans nos états capitalistes, marqués par l'hégémonie de la masculinité. Contrairement à la criminologie traditionnelle qui cherche à identifier les différences entre criminels et non-criminels, leur réflexion met en lumière les points communs entre les femmes justiciables et les autres femmes; c'est une analyse qui, à travers la problématique plus propre aux femmes justiciables, documente la situation de domination, de subordination ou encore différentes dimensions des relations de sexe qui affectent toutes les femmes, à différents titres, dans nos sociétés 
modernes. En effet, même lorsqu'on reconnaît le caractère de classe de l'intervention pénale, les femmes des classes défavorisées n'en maintiennent pas moins leur unité avec l'ensemble des femmes à travers la situation de vie qu'elles partagent toutes comme catégorie de sexe. D'ailleurs, les auteures qui s'inspirent du post-modernisme et refusent de souscrire à l'universalité de la subordination des femmes mettent en cause à leur tour la quête traditionnelle des différences entre les femmes infracteures et l'ensembles des femmes. Worrall (1990), par exemple, présente les multiples formes de contrôle auxquelles les femmes sont soumises à travers le savoir des experts et les multiples moyens de résistance que celles-ci déploient. Elle nous montre comment se construit la féminité qui détermine la vie de l'ensemble des femmes mais cette fois plus particulièrement dans le site pénal.

Dans le cadre de cette approche, le contrôle pénal n'est qu'une facette du contrôle exercé sur les femmes, qu'une instance où se reproduisent leurs conditions d'oppression ou les relations de sexe. D'ailleurs, le faible nombre de femmes prises en charge à ce niveau renvoie à d'autres formes de contrôle (famille, politique sociale, etc.) et indique bien la nécessité de l'articulation des liens entre ces différentes formes de contrôle. La notion de contrevenante ne répond donc pas aux besoins de l'analyse, et les féministes cherchent plutôt à rendre compte des paramètres qui affectent à la fois les femmes justiciables et les autres femmes et à faire ressortir les liens qui les unissent (Eaton, 1986, p. 7). Ces auteures et auteurs examinent les fondements de l'oppression des femmes justiciables ou les paramètres des relations de sexe qui affectent celles-ci en rapport avec l'ensemble des femmes et étendent l'analyse à des thèmes qui affectent leur vie comme la sexualité, le contrôle médical, etc. (Hutter et Williams, 1981 : Smart et Smart, 1978 ; Smart, 1989).

Pour l'ensemble de ceux et celles qui ont adopté cette voie, une analyse féministe de la criminalisation des femmes apparaît donc comme une analyse de l'oppression des femmes ou des relations de sexe telles qu'elles s'exercent en un point donné, soit le système pénal, mais en relation avec les fondements socio-économiques et/ou l'ensemble des relations de sexe qui affectent leur vie. Cette analyse ouvre donc la voie à des études qui ne se confinent pas au cadre pénal mais qui couvrent tant les contrôles informels (la famille, etc.) que les autres variantes du contrôle formel (les politiques sociales, les institutions médicales et psychiatriques, etc.) qui marquent la vie des femmes.

À ce type d'analyse qui vise à mettre en évidence les fondements de l'oppression des femmes et/ou les paramètres des relations de sexe se greffent des propositions de changement. Comme le souligne fort juste- 
ment Currie (1986, p. 242), la réflexion théorique dans une perspective féministe a toujours constitué un processus politique. Aussi, quelle que soit l'approche théorique sous laquelle logent les différents auteurs et auteures, tous et toutes manifestent un engagement pour le changement social, pour la lutte contre l'oppression que subissent les femmes aux prises avec la justice mais aussi les femmes en général.

Parfois, comme le souligne Klein et Kress (1976), un tel engagement prend la forme limitée d'une empathie, d'une préoccupation certaine pour les femmes sans formuler de stratégies précises d'action. Leur analyse n'en est pas moins une mise en cause des approches traditionnelles qui amènent à repenser les politiques pénales qui leur sont reliées.

Mais, dans nombre de réflexions theoriques, les auteures et auteurs joignent explicitement à l'analyse critique des propositions pratiques de changement qui vont de la réorientation des recherches dans le champ criminologique, aux réformes du système de justice criminelle, aux directives pour renouveler le travail des professionnels auprès des femmes justiciables et à la redéfinition des structures de pouvoir dans nos sociétés.

Rafter et Natalazia (1981, p. 85), par exemple, proposent une réorientation des recherches qui permet de mettre en cause les stéréotypes de classe de la "femme idéale" et les fondements patriarcaux de la société. Elles indiquent d'abord la nécessité d'obtenir de meilleurs données sur la «criminalité» des femmes pour étudier plus adéquatement la question. Ensuite, elles mettent en évidence le besoin de documenter largement les contextes sociaux qui entourent le passage à l'acte des femmes et la réaction sociale qui y est associée. Finalement, elles font ressortir l'importance de bien saisir l'attitude du personnel de justice face aux femmes qui entrent en contact avec le pénal, soit comme victimes, comme contrevenantes ou comme employés des institutions pénales.

À cette première dimension de l'action, ajoutons des propositions de réformes du système de justice criminelle (Balkan, Berger, Schmidt, 1980; Carlen, 1988; Dahl et Snare, 1978; Gregory, 1986; Harris, 1987; Heidensohn, 1986; Messerschmidt, 1986: Rafter et Natalazia, 1981). Dahl et Snare (1978), par exemple, mettent l'accent sur la nécessité de réformer la prison afin de permettre aux femmes incarcérées d'assumer leur vie à la fin de leur sentence. Balkan, Berger et Schmidt (1980) proposent un moratoire sur la construction des prisons, un meilleur accès des personnes libres aux institutions carcérales, une lutte 
contre les abus de médication, contre les programmes de modification de comportement, etc. Carlen (1988) et Heidensohn (1986) préconisent une politique pénale axée sur les besoins des femmes et un système carcêral séparé pour ces dernières, en réponse à leurs besoins précis, comprenant des petites maisons ou hôtels, avec de bons liens et soutiens communautaires. Rafter et Natalazia (1981) indiquent la nécessité: a) d'apporter des changements législatifs (par exemple l'abolition des infractions statutaires dans le système de justice des mineurs); $b$ ) de modifier le régime des prisons de façon qu'il ne renforce pas la dépendance des femmes, leur conformité aux rôles sexuels; $c$ ) d'assurer aux hommes et aux femmes l'égalité d'accès à l'emploi comme professionnels dans le domaine de la justice; et $d$ ) de faciliter plus particulièrement cet accès à l'emploi pour les femmes issues de milieux socio-économiques défavorisés. Gregory (1986) met l'accent sur la critique des politiques pénales existantes afin d'en faire ressortir les contradictions et de favoriser les mesures de rechange à l'incarcération et de décriminalisation. Mais, souligne-t-elle, ces mesures doivent être intégrées dans le cadre d'une politique criminelle globale qui tienne compte de toutes les parties impliquées dans les situations problemes, tant les infracteurs, leurs épouses, les enfants que les victimes, etc. La politique pénale alternative doit aussi assurer un traitement humanitaire de tous les cas, que l'on puisse ou non les régler à partir de mesures de décriminalisation ou de rechanges à l'incarcération. Carlen (1990) propose même l'abolition de l'emprisonnement pour les femmes à titre d'expérience pilote pour une durée de cing ans. Selon elle, ce projet pourrait servir de prototype pour l'abolition graduelle de l'emprisonnement comme mesure sentencielle telle que nous la connaissons actuellement. Finalement, des auteures comme Temin (1973), (Harris 1987) et Heidensohn (1986) proposent alors des réformes, la restructuration ou l'abolition du droit pénal.

Nous retrouvons aussi des auteures comme Cain (1989) et Worrall (1990) qui se penchent sur le travail professionnel auprès des femmes justiciables. Worrall (1990), par exemple, suggère aux agents de probation de concentrer leur travail auprès de femmes justiciables sur les services et le soutien dont elles ont besoin pour traverser cette épreuve, plutôt que de tenter de les traiter ou encore de les dominer.

Pour plusieurs auteures et auteurs (Balkan, Berger et Schmidt, 1980; Currie, 1986; Dahl et Snare (1978); Gregory, 1986; Hutter et Williams, 1981 ; Klein et Kress, 1976; Lacombe, 1982 ; Messerschmidt, 1986; Rafter et Natalazia, 1981), ces mesures de réforme doivent faire partie d'une recherche de changements qui touchent aux fondements 
même de la société, qui en attaquent les structures de classe et/ou de «sexe», et/ou de «race». Comme l'indiquent fort justement Rafter et Natalazia (1981), il ne s'agit pas de puiser à des réformes partielles qui ne contribueraient qu'à consolider la structure de classe (et nous pouvons ajouter les rapports sociaux de sexe). Non, Messerschmidt (1986), par exemple, propose un programme qui permet de faire face à certaines formes de comportements de transgression et contribue à modifier la structure actuelle de la société à travers une politique de plein emploi, la démocratisation du lieu de travail et la redéfinition des rapports hommes-femmes dans le cadre de la vie intime. Hutter et Williams (1981) indiquent qu'il faut rejeter les catégorisations imposées aux femmes en ce qu'elles contribuent à faire ressortir leurs différences, à masquer ce qui les unit et à les maintenir sous domination. Les femmes sont des êtres libres, nous rappellent-elles, et peuvent donc se dégager de ces dominations et réclamer leur liberté.

Les travaux féministes sur la criminalisation des femmes ont donc adopté ouvertement une position de réforme en matière pénale, voire des institutions et des structures politiques. Cette orientation nous semble largement tributaire d'une conception de la déviance qui rompt avec la conception traditionnelle. En associant d'emblée les femmes justiciables à l'ensemble des femmes dont elles partagent les conditions générales de vie comme catégorie de sexe, les analyses féministes ont pu s'orienter vers des propositions de changement qui favorisent la modération, et qui partent des besoins et des intérêts des femmes ellesmêmes. Elles ont d'ailleurs réussi, dans certains cas, à attirer l'attention des autorités politiques et/ou judiciaires et correctionnelles. Au Canada, par exemple, les études féministes ont inspiré les responsables du rapport «La Création de Choix» qui proposent une refonte en profondeur du traitement carcéral des femmes condamnées à des sentences de deux ans et plus. Endossé par le gouvernement fédéral, le rapport doit être mis en œuvre et fera la preuve, espérons-le, que le discours féministe, lorsque reconnu par les autorités pénales, peut trouver une application concrète dans les réformes du système de justice criminelle ${ }^{8}$.

8. Pour une analyse de l'influence féministe sur le groupe de travail qui a produit le rapport « La Création de Choix », voir Biron (1992). 


\section{CONCLUSION}

Comme nous avons pu le constater, les analyses féministes sur la "criminalitê" et la criminalisation des femmes contribuent de façon significative au questionnement des approches courantes en criminologie et en politiques criminelles.

D'abord, ces recherches ont introduit la question des femmes dans l'étude de la déviance; elles ont contribué ainsi à dénoncer le caractère partiel et partial (Juteau-Lee, 1981) de la criminologie, et donné les conditions de possibilité pour questionner la conception de la déviance.

Ensuite, elles étudient les liens communs entre les femmes justiciables et les femmes en général plutôt que leurs différences, comme dans le cadre de la criminologie traditionnelle. Le regard porté sur les femmes justiciables échappe donc à la stigmatisation qui accompagne trop souvent les réflexions de la criminologie marquée par le positivisme. En effet, à l'instar des interactionnistes, les féministes présentent des analyses marquées par un respect, une empathie véritable envers les sujets de recherche. Mais, à cette empathie, elles ont également su joindre une préoccupation émancipatoire et se mettre à la recherche de solutions aux problèmes vécus par les femmes.

En outre, portées par le souci de documenter le quotidien des femmes sans ignorer les déterminants structuraux qui marquent leur vie, des études féministes ont pu dépasser le piège réducteur de la dichotomie analyse micro-structurelle, analyse macro-structurelle auquel avaient cédé nombre de travaux critiques en criminologie. On peut donc identifier des études féministes qui portent sur le quotidien des femmes certes, empruntant ainsi à l'interactionnisme, mais qui rendent compte des dimensions de classe, de «sexe» ou de "race» qui marquent leur vie.

On doit également noter que les féministes, dans leur recherche de réponse aux besoins des femmes, n'ont pas reculé devant le défi de considérer à la fois la dimension du comportement et celle de la réaction sociale dans l'analyse. Ce faisant, elles ont permis de concevoir la réaction sociale comme déterminante du comportement et introduit la possibilité d'études qui considèrent les différentes dimensions des situations-problèmes à l'instar de certains travaux des psychologues belges dont Debuyst $(1977 ; 1981 ; 1989)$, François (1979) et Walgrave (1980). 
En ce qui a trait aux solutions proposées, nombre d'auteures adoptent une perspective élargie qui emprunte conjointement aux politiques sociales et pénales. On note ici une reconnaissance de la complexité de la problématique et de la nécessité d'articuler l'action en tenant compte à la fois des besoins immédiats des femmes tant au niveau du système pénal que de leur contexte plus global de vie et de leurs besoins à long terme pour modifier les paramètres qui marquent les relations de sexe et limitent leurs options de vie sociale. Ces études dépassent donc le problème associé au silence, à la non-intervention radicale ou encore aux seules propositions de «réformes négatives» de nombre d'analyses du contrôle pénal présentées par des criminologues critiques. À l'instar du premier moment de l'École de Chicago, elles proposent des réformes sociales; mais à la suite des interactionnistes de la réaction sociale, elles proposent aussi des réformes du système pénal axées sur le principe de modération, dont des mesures de décriminalisation ou de dépénalisation.

Dans l'ensemble, il nous apparaît que le souci féministe de répondre aux besoins et aux intérêts des femmes comme groupe leur a donné les conditions pour dépasser plusieurs limites qui ont marqué les perspectives masculines de la discipline. Leurs recherches ouvrent donc de nouvelle voies qui peuvent servir d'inspiration au questionnement actuel dans la criminologie. Chose certaine, aujourd'hui, l'béritage féministe semble assez important et diversifié pour affirmer que la discipline criminologique a besoin du féminisme. Mais faudrait-il encore que cet apport soit enfin reconnu.

\section{BIBLIOGRAPHIE}

ADLER, F. (1975), Sisters in Crime. The Rise of the New Female Criminal, New York, McGraw-Hill.

BALKAN, S., BERGER, R. J., SCHMIDT, J. (1980), Crime and Deviance in America: A Critical Approach, Belmont, Cal., Wadsworth Pub. Co.

BEAUVOIR DE, S., (1949), Le deuxième sexe, Paris, Gallimard.

BERTRAND, M. A., (1979), La femme et le crime, Montréal, L'Aurore.

BIRON, L. L. «Les femmes el l'incarcération, le temps n'arrange nien», Criminologie, vol. XXV, no 1, 1992, p. 119-134. 
BLOM, M., VAN DEN BERG, T., (1989), «A typology of the life and work styles of "heroin-prostitutes" ", in M. Cain (Ed.), Growing Up Good. Policing the Behaviour of Girls in Europe, (p. 55-69), London, Sage.

BOX, S., (1983), Power, Crime and Mystification, New York Tavistock.

CAIN, M., «Towards Transgression: New Directions in Feminist Criminology », International Journal of Sociology of Law, vol. 18, 1990, p. 1-18.

CANADA, (1990), La Création de Choix, rapport du groupe d'étude sur les femmes purgeant une sentence fédérale, Ottawa.

CARLEN, P. (êd.), (1985), Criminal Women. Autobiographical Accounts, Cambridge, Polity Press.

CARLEN, P., (1988), Women, Crime and Poverty, Milton Keynes, Philadelphia, Open University Press.

CARLEN, P., «Ferninist Jurisprudence-or Women-Wise Penology?», Probation Journal, vol. $36, \mathrm{n}^{\circ} 3,1989$, p. 110-114.

CARLEN, P., (1990), Alternative to Women's Imprisonment, Milton Keynes, Open University Press.

CURRIE, D., (1986), «Female Criminality: A Crisis in Feminist Theory $*$, in B.D. Mac Lean ( $(\mathrm{d}$.), The Political Economy of Crime, (p. 232-246), Scarborough, PrenticeHall, Canada.

DAHL, T. S., SNARE, A., "The coercion of privacy. A feminist perspective , in C. Smart et B. Smart (éds.), Women, Sexuality and Social Control, (p. 8-26), London, Routledge and Kegan Paul.

DALY, K., * Discrimination in the Criminal Court: Family, Gender, and the Problem of Equal Treatment $»$, Social Forces, vol. 66, $\mathrm{n}^{\circ} 1,1987$, p. 152-175.

DEBUYST, C., «Le concept de dangerosité et un de ses élếments constitutifs: la personnalité (criminelle)», déviance et Société, vol. 1, nº 4, 1977, p. 363-387.

DEBUYST, C., (1981), «Le concept de personnalité dangereuse considéré comme expression d'un point de vue " in C. Debuyst (éd.), Dangerosité et justice pénale, (p. 19-34), Genève, Masson-Médecine et Hygiène.

DEBUYST, $C$., «Perspectives cliniques en criminologie. Le choix d'une orientation», Revue internationale de criminologie et de police technique, vol. 4, 1989, p. 405418.

EATON, M., (1986), Justice for Women? Family, Court and Social Control, Milton Keynes, Philadelphia, Open University Press.

FRANÇOIS J., «Pour une clinique alternative en milieu carcéral », Déviance et Société, vol. $3, n^{\circ} 2,1979$, p. 169-178.

GELSTHORPE, L., MORRIS, A., «Feminism and Criminology in Britain ", British Journal of Criminology, vol. $28, n^{\circ} 2,1988$, p. 224-240.

GELSTHORPE, L., MORRIS, A., (1990), Feminist Perspectives in Criminology, Milton Keynes, Open University Press. 
GREGORY, J., (1986), « Sex, class and crime: towards a non-sexist criminology », in R. Matthews et J. Young (eds.), Confronting Crime, (p. 53-71), Beverly Hills, Sage.

HARRIS, M.K., "Moving into the New Millenium: Toward a Feminist Vision of Justice ${ }$, The Prison Journal, vol. 67, 1987, p. 27-38.

HEIDENSOHN, F., "The Deviance of Woman: a Critique and an Enquiry », British Journal of Sociology, vol. 19, $n^{\circ} 2,1968$, p. 160-176.

HEIDENSOHN, F., (1985), Women and Crime, New York, University Press.

HEIDENSOHN, F., «Models of Justice: Portia or Persephone? Some Thoughts on Equality, Faimess and Gender in the Field of Criminal Justice *, International Journal of Sociology of Law, vol. 14, 1986, p. 287-298.

HUTTER, B., WILliAMS, G. (éd.), (1981), Controlling Women. The Normal and the Deviant, London: Crom Helm London and the Oxford University Women's Studies Committee.

JUTEAU-LEE, D., (1981), "Visions partielles, visions partiales : visions (des) minoritaires en sociologie ", Sociologie et Sociétés, vol. XIII, n² 2, p. 33-47.

KLEIN D., "The Etiology of female Crime: A Review of the Literature», Issues in Criminology, vol. 8, $\mathrm{n}^{\circ} 2,1973$, p. 3-30.

KLEIN, D., KRESS, J., * Any woman's Blues : A Critical Overview of Women, Crime and the Criminal Justice System m, Crime and Social Justice, vol. 5, 1976, p. 3447.

LABERGE, D., «Women's Criminality, Criminal Women, Criminalized Women? Questions In and For a Feminist Perspective», The Journal of Human Justice, vol. $2, n^{\circ} 2,1991$, p. 37-56.

LACOMBE, D., «Two Views on the Oppression of Women: The Limitations of Marxist and Radical Feminist Perspectives », Canadian Criminology Forum, vol. 6, 1984 , p. $165-176$.

LAPRAIRIE, C., (1987), "Native Women and Crime in Canada: A Theoretical Model », in E. Adelberg, C. Currie (éds.), Too Few To Count. Canadian Women in Conflict with the Law, (p. 103-112), Vancouver, Press Gang Pub.

LEONARD, E. B., (1982), Women, Crime and Society, New York, Longman.

MESSERSCHMIDT, J. W., (1986), Capitalism, Patriarchy and Crime: Toward a Socialist Feminist Criminology, Totowa, New Jersey: Rowman and Littlefield.

NAFFINE, N., (1987), Female Crime: The Construction of Women in Criminology, Sydney, Allen and Unwin.

PARENT, C., «La protection chevaleresque ou les representations masculines du traitement des femmes dans la justice pénale », Déviance et Société, vol. 10, $\mathbf{n}^{\circ} 2,1986$, p 147-175.

PARENT, C., «Au-delà du silence: les productions féministes sur la criminalité et la criminalisation des femmes ", Déviance et Société, vol. 16, $\mathrm{n}^{\circ} 3,1992$, p. 298-328. 
RAFTER, N. H., NATALAZIA, E. M., «Marxist Feminism-Implications for Criminal Justice », Crime and Delinquency, vol. 27, 1981, p. 81-99.

RAINS, P., (1971), Becoming an Unwed Mother, Chicago, Adline-Atherton.

SCHUR, E.M., (1984), Labeling Women Deviant. Gender, Stigma and Social Control, Philadelphia, Temple University Press.

SCHWENDINGER, H. et J., * Rape Myths: In Legal, Theoretical, and Everyday Practice ", Crime and Social Justice, vol. 1, 1974, p. 18-26.

SIMON, R.J., (1975), Women and Crime, London, Lexington.

SMART, C., (1976), Women. Crime and Criminology. A Feminist Critique, London, Routledge and Kegan Paul.

SMART, C., "The New Female Criminal: Reality or Myth?», British Journal of Criminology, vol. $19, \mathrm{n}^{\circ} 1,1979$, p. $50-59$.

SMART. C., «Feminism and Law: Some Problems of Analysis and Strategy", International Journal of Sociology of Law, vol. 14, 1986, p. 109-123.

SMART, C.. (1989), Feminism and the Power of Law, New York, Routledge.

SMART, C., (1990), «Feminist approaches to criminology or postmodern woman meets atavistic man », in L. Gelsthorpe et A. Morris (éds.), Feminist Perspectives in Criminology, (p. 70-84), Milton Keynes, Open University Press.

SMART, B., SMART, C., (1978), "Women and Social Control", in B. Smart et C. Smart (eds.), Women, Sexuality and Social Control, (p. 1-7), Boston, Routledge and Kegan Paul.

SUMNER, C., (1990), «Foucault, gender and the censure of deviance», in L. Gelsthorpe and A. Morris (éds.), Feminist Perspectives in Criminology, (p. 26-40), Milton Keynes, Open University Press.

TEMIN, C.E., (1973), "Discriminatory Sentencing of Women Offenders; The Argument for ERA in a Nutshell $m$, in S. K. Datesman et F. R. Scarpitti (eds.), (1980), Women, Crime and Justice, (p. 255-276), New York, Oxford University Press.

THOMAS, W. I., (1923), The Unadjusted Girl, New York, Harper and Row.

WALGRAVE, L., (1980), «Considérations sur l'orientation de la psychologie dans la criminologie actuelle », Déviance et Société, vol. 4, n 4, p. 305-330.

WORRALL, A., (1990), Offending Women. Female Lawbreakers and the Criminal Justice System, New York, Routledge. 